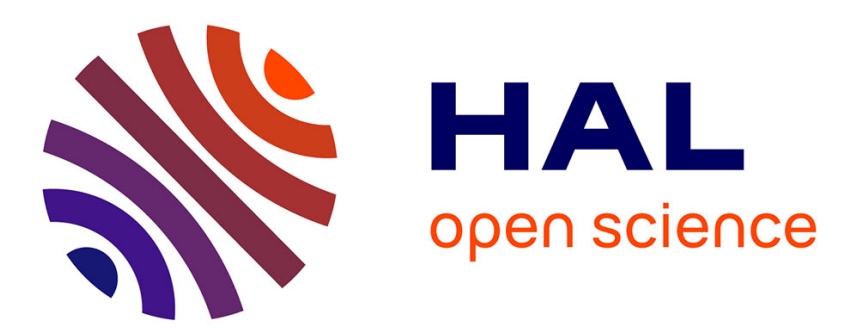

\title{
Shape coexistence and phase transitions in the platinum isotopes
}

\author{
I.O. Morales, A. Frank, C.E. Vargas, P. van Isacker
}

\section{To cite this version:}

I.O. Morales, A. Frank, C.E. Vargas, P. van Isacker. Shape coexistence and phase transitions in the platinum isotopes. Physical Review C, 2008, 78, pp.024303. 10.1103/PhysRevC.78.024303 . in2p300311179

\section{HAL Id: in2p3-00311179 \\ https://hal.in2p3.fr/in2p3-00311179}

Submitted on 13 Aug 2008

HAL is a multi-disciplinary open access archive for the deposit and dissemination of scientific research documents, whether they are published or not. The documents may come from teaching and research institutions in France or abroad, or from public or private research centers.
L'archive ouverte pluridisciplinaire HAL, est destinée au dépôt et à la diffusion de documents scientifiques de niveau recherche, publiés ou non, émanant des établissements d'enseignement et de recherche français ou étrangers, des laboratoires publics ou privés. 


\title{
Shape coexistence and phase transitions in the platinum isotopes
}

\author{
Irving O. Morales \\ Instituto de Ciencias Nucleares, UNAM, Apdo. Postal 70-543, 04510 México, D.F. México \\ Alejandro Frank \\ Instituto de Ciencias Nucleares, UNAM, Apdo. Postal 70-543, 04510 México, D.F. México \\ Carlos E. Vargas \\ Facultad de Física e Inteligencia Artificial, Universidad Veracruzana \\ Sebastián Camacho 5; Centro, Xalapa, Ver., 91000, México \\ P. Van Isacker \\ Grand Accélérateur National d'Ions Lourds CEA/DSM-CNRS/IN2P3, B.P. 55027, F-14076 Caen Cedex 5, France
}

(Dated: February 26, 2008)

\begin{abstract}
The matrix coherent-state approach of the interacting boson model with configuration mixing is used to study the geometry of the platinum isotopes. With a parameter set determined in previous studies, it is found that the absolute minimum of the potential for the $\mathrm{Pt}$ isotopes evolves from spherical to oblate and finally to prolate shapes when the neutron number decreases from $N=126$ (semi-magic) to $N=104$ (mid-shell). Shape coexistence is found in the isotopes ${ }^{182,184,186,188} \mathrm{Pt}$. A phase diagram is constructed which shows the coexistence region as a function of the number of bosons and the strength of the mixing parameter.
\end{abstract}

PACS numbers: 21.10.Re, 21.60.Ev, 21.60.Fw, 27.70.+q

\section{INTRODUCTION}

The phenomenon of shape coexistence in nuclei has been studied for decades and still is an active topic of research. Conversion electron coincidence, recoil and recoil-decay tagging techniques, heavy-ion induced fusion-evaporation reactions, and the study of the finestructure of $\alpha$ decay have, for example, allowed the identification of three low-lying $0^{+}$states in ${ }^{186} \mathrm{~Pb}$ [1], each interpreted in terms of a distinct shape, originating from multiple particle-hole excitations across the $Z=82$ proton shell gap. Coexisting shapes have also been proposed to influence the yrast states in ${ }^{174} \mathrm{Pt}$ [2] where mixing between different nuclear configurations results in the observation of a single mixed yrast band. Investigations in ${ }^{176-182} \mathrm{Pt}$ have suggested that the two lowest-lying $0^{+}$of these isotopes are formed from the mixing of two intrinsic states of different deformation [3].

Calculations within a deformed mean-field approximation have indicated the possibility to find close-lying oblate and prolate minima next to the spherical groundstate configuration of ${ }^{186} \mathrm{~Pb}[4]$. In general, these studies have focussed on the properties of static potential energy surfaces and only recently it has become possible to implement the generator coordinate method with symmetry restoration which yields spectroscopic properties of nuclei, albeit with considerable numerical effort (for an example in the neutron-deficient $\mathrm{Pb}$ isotopes, see Ref. [5]). Shell-model studies [6] of the coexistence phenomenon are even more numerically challenging due to the large spaces involved. The Interacting Boson Model (IBM) [7, 8], on the other hand, assumes a truncated shell-model Hilbert space composed of correlated like- nucleon pairs coupled to angular momentum $L=0$ and 2 , which are subsequently approximated as bosons, and employs an appropiate Hamiltonian in this space. This simplified analysis makes possible a calculation consistent with both the spectroscopic properties and geometry of an isotope series. In particular, for the Pt isotopes a transitional Hamiltonian can be proposed which covers the range from $\mathrm{U}(5)$ vibrational to $\mathrm{SU}(3)$ rotational nuclei.

The use of coherent states allows one to derive a potential energy surface starting from an algebraic IBM Hamiltonian, which can in turn be used to determine the equilibrium configurations of the system and their corresponding shapes in terms of the $\beta$ and $\gamma$ variables. In case there are close-lying configurations which can mix, the appropriate algebraic framework was first proposed by Duval and Barrett [9]. Their approach leads to coexisting minima that can be associated to the presence of intruder excitations $[10,11]$, in particular at or near shell closures. The origin of these intruder states can be traced back to many-particle many-hole ( $\mathrm{p}-\mathrm{h})$ excitations across shell gaps [12]. Duval and Barrett [9] suggested the possibility of including the simplest intruder $2 \mathrm{p}-2 \mathrm{~h}$ configuration by adding two extra bosons to the IBM, and allowing this configuration to mix with the regular (ground-state) one with $N$ bosons. In the $\mathrm{Pb}$ isotopes, where a coexistence of spherical, oblate and prolate shapes seems to occur (in particular in ${ }^{186} \mathrm{~Pb}$ ), three configurations are required of a regular, $2 \mathrm{p}-2 \mathrm{~h}$, and $4 \mathrm{p}-$ 4h type. The configuration-mixing IBM has allowed a simultaneous description of the whole series of $\mathrm{Pb}$ isotopes with a single Hamiltonian [13]. To analyze the geometry of such Hamiltonians, a matrix coherent-state 
method was introduced $[14,15]$ which allows a consistent description of shape coexistence phenomena, as well as an analysis of the phase diagrams and the transitional behavior of IBM Hamiltonians [16, 17].

In this paper we use the matrix coherent-state method to study the evolution of the shape and the phenomenon of coexistence in the $\mathrm{Pt}$ isotopes and we present the phase diagrams associated to the chain. The paper is organized as follows. In Sect. II the model Hamiltonian and the matrix formulation of the problem are introduced. The results for the shape potentials and phase diagrams in the ${ }^{182-198} \mathrm{Pt}$ isotopes are presented in Sects. III and IV. A brief summary and conclusions are given in Sect. V.

\section{THE MODEL HAMILTONIAN}

The Hamiltonian of the configuration-mixing IBM is

$$
\hat{H}=\hat{H}_{0 \mathrm{p}-0 \mathrm{~h}}+\hat{H}_{2 \mathrm{p}-2 \mathrm{~h}}+\hat{H}_{\mathrm{mix}} .
$$

This Hamiltonian acts in a Hilbert space which consists of the sum of two symmetric $\mathrm{U}(6)$ representations $[N] \oplus$ $[N+2]$, corresponding to the $0 \mathrm{p}-0 \mathrm{~h}$ and $2 \mathrm{p}-2 \mathrm{~h}$ excitations, respectively. The separate pieces of the Hamiltonian are

$$
\hat{H}_{i}=\epsilon_{i} \hat{n}_{d}+\kappa_{i} \hat{Q}_{i} \cdot \hat{Q}_{i}+\kappa_{i}^{\prime} \hat{L} \cdot \hat{L}
$$

where $\hat{H}_{i}(i=0$ or 2$)$ are the Hamiltonian operators acting in the $0 \mathrm{p}-0 \mathrm{~h}$ (regular) and $2 \mathrm{p}-2 \mathrm{~h}$ (deformed) subspaces, respectively. Furthermore, the operator $\hat{n}_{d}$ counts the number of $d$ bosons, $\hat{L}$ is the angular momentum operator, $\hat{Q}_{i}$ is the standard quadrupole operator of the
IBM,

$$
\hat{Q}_{i, \mu}=\left(s^{\dagger} \tilde{d}+d^{\dagger} \tilde{s}\right)_{\mu}^{(2)}+\chi_{i}\left(d^{\dagger} \tilde{d}\right)_{\mu}^{(2)},
$$

and $\hat{H}_{\text {mix }}$ mixes the two configurations,

$$
\hat{H}_{\mathrm{mix}}=\omega\left[\left(s^{\dagger} s^{\dagger}+\tilde{s} \tilde{s}\right)^{(0)}+\left(d^{\dagger} d^{\dagger}+\tilde{d} \tilde{d}\right)^{(0)}\right] .
$$

The parameters $\epsilon_{i}, \kappa_{i}$ and $\chi_{i}$ in Eqs. (2) and (3) pertain to the $0 \mathrm{p}-0 \mathrm{~h}(i=0)$ and $2 \mathrm{p}-2 \mathrm{~h}(i=2)$ configurations, and have been taken from Ref. [18] without any modification. These parameters were extracted from a comprehensive fit to excitation energies and $B(\mathrm{E} 2)$ values in the ${ }^{194} \mathrm{Pt}$ and ${ }^{196} \mathrm{Pt}$ isotopes for the regular $N$ configuration and, based on the concept of $I$-spin symmetry [19], from the W isotopes for the $2 \mathrm{p}-2 \mathrm{~h} N+2$ configuration. The intensity of the mixing is determined by the parameter $\omega$. We have taken for the calculation of potential energy surfaces the average value $\omega=50 \mathrm{keV}$ but for the phase diagram a larger range has been considered in order to map the complete region.

The algebraic formalism does not provide directly a geometric interpretation in terms of shapes but this can be achieved with the theory of coherent states [20]. A geometric interpretation is obtained by computing the expectation value of the Hamiltonian in the ground coherent state, a procedure which is known as the classical limit $[21,22]$. Hence a connection was established between the IBM and the Bohr-Mottelson geometrical model [23], giving an intrinsic geometric structure to the former. With the coherent-state formalism the following energy surface is obtained for a general IBM Hamiltonian

$$
E(N, \beta, \gamma)=a_{1}^{(0)}+\frac{N}{1+\beta^{2}}\left(a_{1}^{(1)} \beta^{2}+a_{2}^{(1)}\right)+\frac{N(N-1)}{\left(1+\beta^{2}\right)^{2}}\left(a_{1}^{(2)} \beta^{4}+a_{2}^{(2)} \beta^{3} \cos 3 \gamma+a_{3}^{(2)} \beta^{2}+a_{4}^{(2)}\right)
$$

where the $a_{i}^{(k)}$ are fixed in terms of the parameters in the Hamiltonian. For the Hamiltonian (2) the energy surface $E_{0}(N, \beta, \gamma)$ in the $0 \mathrm{p}-0 \mathrm{~h}$ configuration is given by

$$
E_{i}(N, \beta, \gamma)=\epsilon_{i} \frac{N}{1+\beta^{2}} \beta^{2}+\kappa_{i}\left[\frac{N}{1+\beta^{2}}\left(5+\left(1+\chi_{i}^{2}\right) \beta^{2}\right)+\frac{N(N-1)}{\left(1+\beta^{2}\right)^{2}}\left(\frac{2}{7} \chi_{i}^{2} \beta^{4}-4 \sqrt{\frac{2}{7}} \chi_{i} \beta^{3} \cos 3 \gamma+4 \beta^{2}\right)\right],
$$

with $i=0$. The classical limit of the Hamiltonian for the $2 \mathrm{p}-2 \mathrm{~h}$ configuration has the same form but with $N \rightarrow N+2$ and $i=2$. The non-diagonal matrix elements $\Omega(\beta)$ are given by the matrix elements of the mixing Hamiltonian (4) between the ground coherent state, leading to

$$
\Omega(\beta)=\frac{\sqrt{(N+2)(N+1)}}{1+\beta^{2}}\left(\omega+\frac{\omega}{\sqrt{5}} \beta^{2}\right) .
$$

For the platinum isotopes, in particular for ${ }^{182-204} \mathrm{Pt}$, a single set of parameters describes their potential energy surfaces for $N$ ranging from 2 to 13 bosons (two proton boson holes and the neutron boson holes counting from the closed shell $N=126$ to midshell at $N=104$ ). The $0 \mathrm{p}-0 \mathrm{~h}$ configuration corresponds to $N$ bosons whereas the $2 \mathrm{p}-2 \mathrm{~h}$ excitations require two extra bosons [9]. This leads to a $2 \times 2$ potential energy matrix $[14,15]$ :

$$
E(\beta, \gamma)=\left(\begin{array}{cc}
E_{0}(N, \beta, \gamma) & \Omega(\beta) \\
\Omega(\beta) & E_{2}(N+2, \beta, \gamma)+\Delta
\end{array}\right)
$$




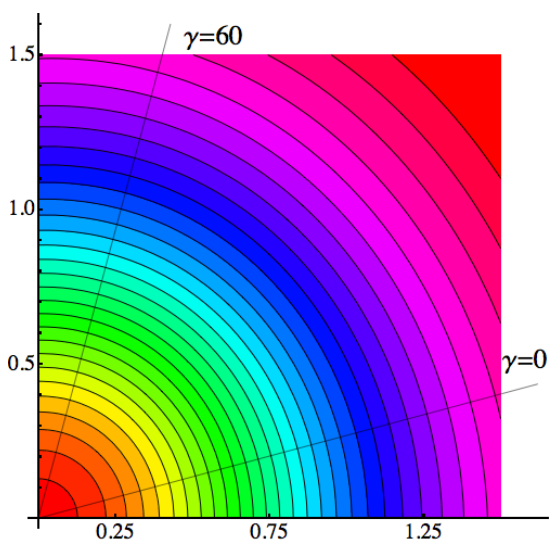

(a)

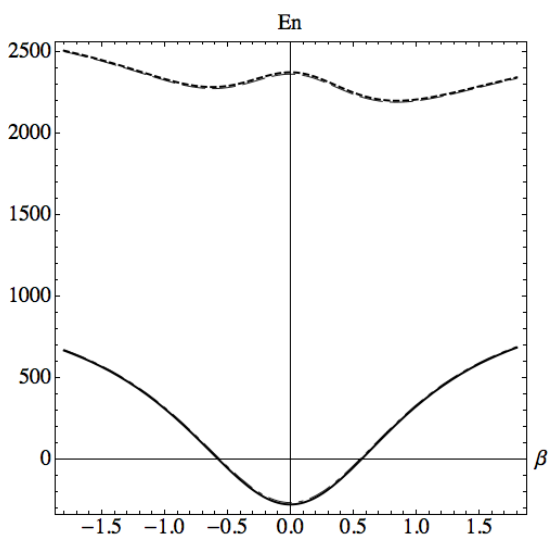

(b)

FIG. 1: Potential energy for ${ }^{204} \mathrm{Pt}$. The upper part shows the lowest eigenpotential of the matrix $E(\beta, \gamma)$ in Eq. (8). The lower part shows the potential for $\gamma=0^{\circ}$ as a function of $\beta$ for the regular (solid line) and $2 \mathrm{p}-2 \mathrm{~h}$ (dot-dashed line) configurations.

where $\Delta$ corresponds to the single-particle energy expended in raising two protons from the lower (50-82) to the upper (82-126) shell, corrected for the gain in energy due to pairing. After numerical diagonalization of this matrix we obtain the eigenpotentials which are presented in the next section.

\section{POTENTIAL ENERGY SURFACES}

In this section we present the potential energy surfaces (PES) for several platinum isotopes from $A=182$ to 204 ; the phase diagram for the entire chain is presented in the next section. Beginning with the heavier isotopes, we show in Fig. 1 the energy surface corresponding lowest eigenpotential for the semi-magic nucleus ${ }^{204} \mathrm{Pt}$. The PES is almost identical to the one of the regular configuration and exhibits a completely spherical shape. The $2 \mathrm{p}-2 \mathrm{~h}$ configuration has a prolate minimum but being more than $2.5 \mathrm{MeV}$ higher in energy, it has a negligi-

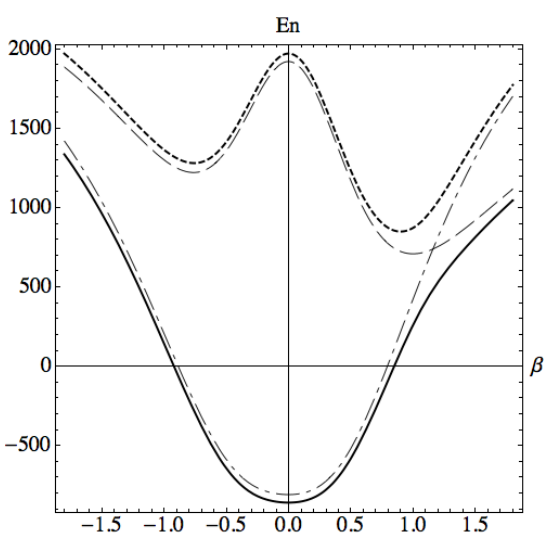

FIG. 2: Potential energy for $\gamma=0^{\circ}$ as a function of $\beta$ for ${ }^{196} \mathrm{Pt}$ for the regular (dot-dashed line) and $2 \mathrm{p}-2 \mathrm{~h}$ (dashed line) configurations, and for the lowest (solid line) and highest (dotted line) mixed configurations.

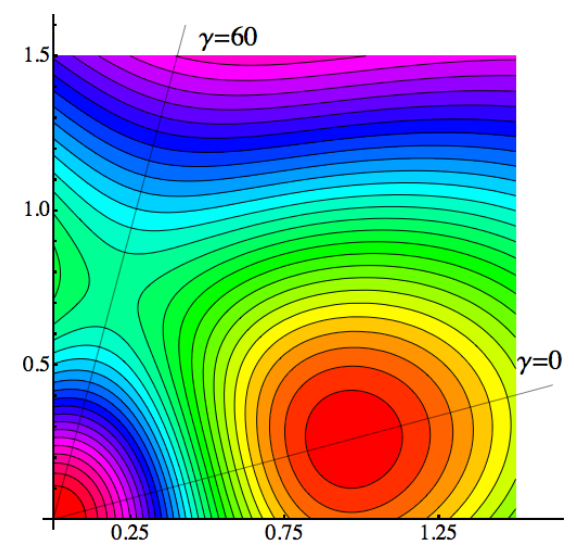

FIG. 3: Potential energy surface in the $\beta-\gamma$ plane for the $2 \mathrm{p}-2 \mathrm{~h}$ configuration in ${ }^{196} \mathrm{Pt}$.

ble contribution to the mixed (eigen)surface. The large energy difference between these configurations which is found in ${ }^{204} \mathrm{Pt}$, decreases when moving towards the midshell isotopes. We also observe that the PES minima associated with the $0 \mathrm{p}-0 \mathrm{~h}$ and $2 \mathrm{p}-2 \mathrm{~h}$ configurations become deeper when moving towards the lighter isotopes as well as closer in energy. This can be seen in Fig. 2 which displays the PES associated with ${ }^{196} \mathrm{Pt}$. The figure shows two apparent minima in the $2 \mathrm{p}-2 \mathrm{~h}$ configuration, but in the $\beta-\gamma$ plot of Fig. 3 it is seen that only one (namely the one with $\gamma=0$ ) is a real minimum while the other extremum, with $\beta<0$, is in fact a saddle point. This analysis shows that each configuration contributes with one minimum and that, if the surfaces are close in energy, their mixing can generate a single surface exhibiting more than one minimum, in principle allowing the phenomenon of coexistence.

The ${ }^{194} \mathrm{Pt}$ isotope, corresponding to 7 bosons, has an almost spherical minimum but shows a tendency to be flat, as can be seen in Fig. 4 . The $2 \mathrm{p}-2 \mathrm{~h}$ potential ex- 


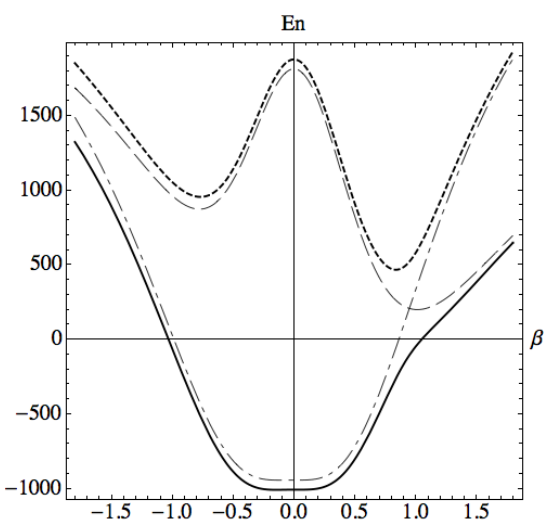

FIG. 4: Potential energy for $\gamma=0^{\circ}$ as a function of $\beta$ for ${ }^{194} \mathrm{Pt}$. Lines are as in Fig. 2.

hibits two extrema in the $\gamma=0$ projection but as in ${ }^{196} \mathrm{Pt}$ the one corresponding to oblate deformation is a saddle point. The ${ }^{190-194} \mathrm{Pt}$ isotopes have a PES flatter than those of nuclei closer to semi-magic ${ }^{204} \mathrm{Pt}$, leading to a region of shapes with apparent $\gamma$ instability. This feature is reminiscent of the $\mathrm{E}(5)$ "critical point symmetry" recently proposed to describe critical behavior [24, 25].

In this region and going towards the lighter isotopes, we find that the flat potential displays a small depression, making them slightly oblate. The nucleus ${ }^{192} \mathrm{Pt}$ is a good example of this feature, as seen in Fig. 5. The absolute oblate minimum at $\gamma=60^{\circ}$, in the upper part of the figure, at $\beta=0.349$ is only $32 \mathrm{keV}$ below the second minimum associated to a spherical shape (lower part of the figure). This very flat potential of the $0 \mathrm{p}-0 \mathrm{~h}$ configuration combined with the one corresponding to $2 \mathrm{p}-2 \mathrm{~h}$, lowered in energy due to pairing, makes the resultant PES nearly $\gamma$ unstable. Figure 6 makes the near- $\gamma$-instability evident in case a stronger mixing is taken, $\omega=200 \mathrm{keV}$. Near- $\gamma-$ instability is also found in ${ }^{186} \mathrm{Pt}$ (11 bosons), where the potential has a minimum at $\beta=0.683$ (see Fig. 7 ). The phase diagram presented in the next section shows that there is indeed a large region in the $\omega-N$ space which corresponds to near $\gamma$-instability.

The PES for ${ }^{188} \mathrm{Pt}$ is shown in Fig. 8. We see that in this case the absolute minimum corresponds to an oblate shape. The nucleus ${ }^{188} \mathrm{Pt}$ has a potential which is deeper and not as flat as the one for heavier isotopes. The oblate deformation arises from the regular configuration while the $2 \mathrm{p}-2 \mathrm{~h}$ configuration is almost $50 \mathrm{keV}$ higher in energy. The mixing makes that difference smaller but still the lowest eigenvalue corresponds to an oblate shape.

In isotopes lighter than ${ }^{188} \mathrm{Pt}$ the PES shows two coexisting minima. In ${ }^{186} \mathrm{Pt}$ the absolute minimum with oblate deformation is nearly $200 \mathrm{keV}$ deeper than the prolate one while in ${ }^{184} \mathrm{Pt}$ the absolute minimum is prolate, being $500 \mathrm{keV}$ deeper than the oblate one. The isotope ${ }^{182} \mathrm{Pt}$ has a potential with a prolate absolute prolate and a second oblate minimum which is $800 \mathrm{keV}$ higher in energy. We show Fig. 9 as a representative example of this

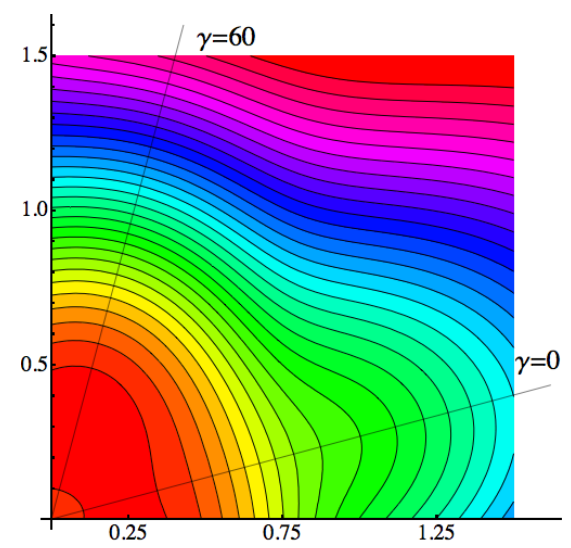

(a)

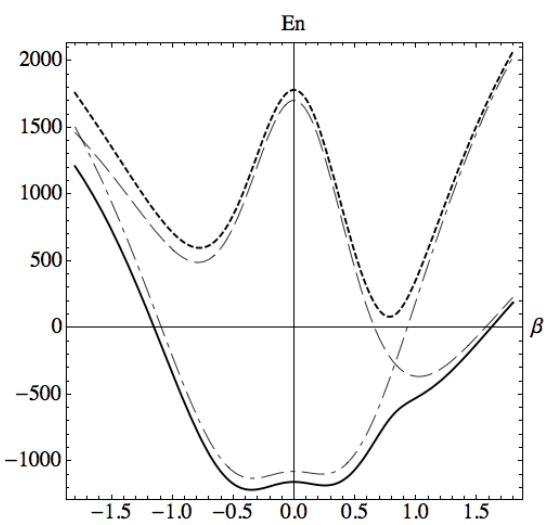

(b)

FIG. 5: Potential energy for ${ }^{192} \mathrm{Pt}$. The upper part shows the lowest eigenpotential of the matrix $E(\beta, \gamma)$ in Eq. (8). The lower part shows the potential for $\gamma=0^{\circ}$ as a function of $\beta$. Lines are as in Fig. 2 .

kind of coexistence, displaying the PES of ${ }^{182} \mathrm{Pt}$. Level curves clearly show both minima and the projection in $\gamma=0^{\circ}$ displays the difference in energy between them.

The predominance of a spherical shape in isotopes heavier than ${ }^{190} \mathrm{Pt}$ seems clear from the PES. The geometrical analysis exhibits the way in which shape coexistence, present in ${ }^{182-188} \mathrm{Pt}$, disappears as the number of neutrons increases. Isotopes heavier than ${ }^{188} \mathrm{Pt}$ have a very flat potential, which is characteristic of this region, as was first demonstrated by Davidson et al. [3], Stuchbery et al. [26], and Bengtsson et al. [27].

A more detailed analysis of the shape coexistence region and the different phases for the $\mathrm{Pt}$ chain is presented in the next section.

\section{PHASE DIAGRAM}

The phase diagram for the $\mathrm{Pt}$ isotopes is shown in Fig. 10. We plot the strength of the mixing parameter $\omega$ of Eq. (4) on the vertical axis against the boson number $N$ on the horizontal axis. These are considered as con- 


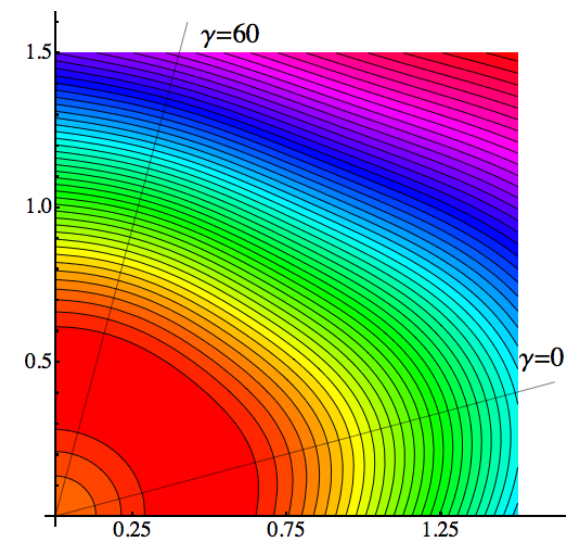

FIG. 6: Potential energy in the $\beta-\gamma$ plane for ${ }^{190} \mathrm{Pt}$ with a strong mixing $\omega=200 \mathrm{keV}$.

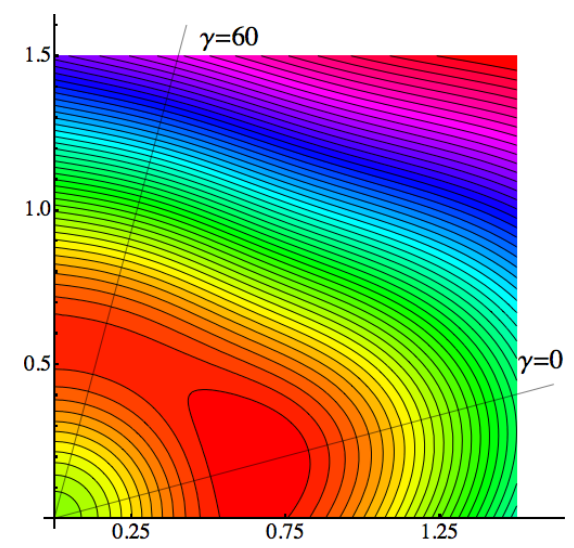

FIG. 7: Potential energy plot in the $\beta-\gamma$ plane for ${ }^{186} \mathrm{Pt}$ with a strong mixing $\omega=200 \mathrm{keV}$.

trol parameters with the boson number, for the sake of clarity, taken as continuous. In the application to the $\mathrm{Pt}$ isotopes of Ref. [18] the mixing parameter $\omega$ was set to values between 0 and $50 \mathrm{keV}$. In order to map a larger region, this parameter is varied between 0 and $400 \mathrm{keV}$ in the present work.

In the left-most region of Fig. 10 the absolute minimum is spherical and the PES is completely dominated by the $0 \mathrm{p}-0 \mathrm{~h}$ configuration. By increasing the number of bosons, this configuration turns oblate while the $2 \mathrm{p}-2 \mathrm{~h}$ configuration goes down in energy. If the difference in energy between the two configurations is small, a region of shape coexistence results, first with the $0 \mathrm{p}-0 \mathrm{~h}$ configuration lowest (oblate-prolate coexistence) and for bigger $N$ with a dominant $2 \mathrm{p}-2 \mathrm{~h}$ configuration (prolate-oblate coexistence). Isotopes with a single prolate minimum are only possible for big mixing values $(\omega>200 \mathrm{keV})$. Around the (Maxwell) line separating the oblate-prolate and prolate-oblate coexistence regions, the two minima have almost the same energy. This corresponds to a $\gamma$ instability zone.

In a very recent paper [17] it has been shown that in

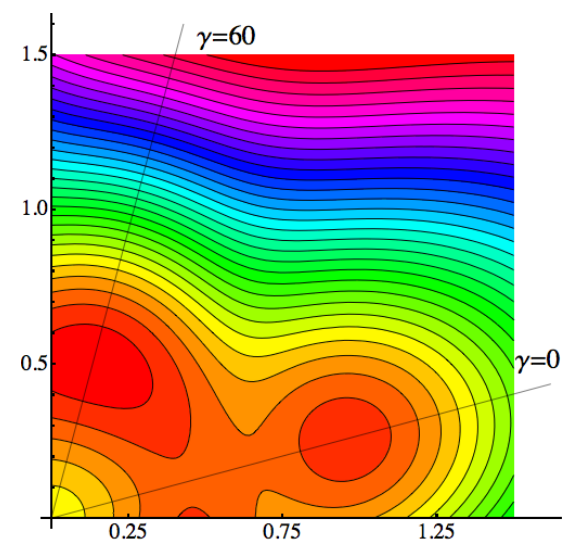

(a)

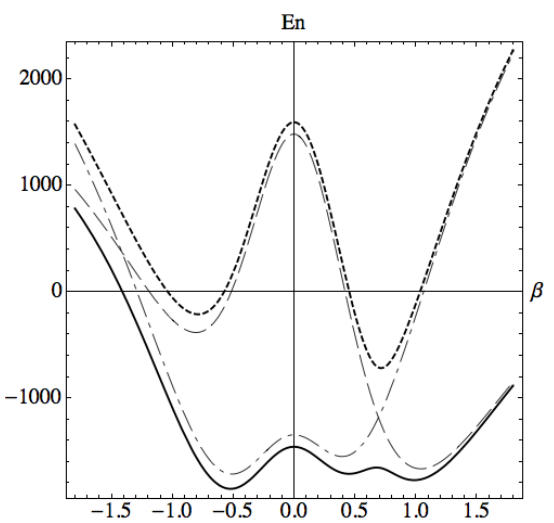

(b)

FIG. 8: Potential energy for ${ }^{188} \mathrm{Pt}$. The upper part shows the lowest eigenpotential of the matrix $E(\beta, \gamma)$ in Eq. (8). The lower part shows the potential for $\gamma=0^{\circ}$ as a function of $\beta$. Lines are as in Fig. 2 .

the IBM with configuration mixing there are large regions of shape coexistence. That work was carried out for mixing between the exact $\mathrm{U}(5)$ limit $(\kappa=0)$ and the exact $\mathrm{SO}(6)$ or $\mathrm{SU}(3)$ limits (i.e., $\epsilon=0$ and either $\chi=0$ or $\chi=\sqrt{7} / 2$ ). As a result coexistence of spherical and deformed shapes was found. In the present study we have not taken these schematic parameters but used realistic values as obtained from a fit to the overall properties of the $\mathrm{Pt}$ isotopes. In particular, since in this parametrization the $0 \mathrm{p}-0 \mathrm{~h}$ configuration turns slightly oblate for large $N$ while the $2 \mathrm{p}-2 \mathrm{~h}$ configuration is prolate deformed, oblate-prolate coexistence becomes possible and is indeed found for realistic values of the mixing parameter $\omega$. The possibility of oblate-prolate coexistence (and its associated $\gamma$ instability) was considered by Hellemans [28] in the context of a schematic model of $\mathrm{SU}_{-}(3)-\mathrm{SU}_{+}(3) \mathrm{mix}-$ ing, and the present results are an excellent example of this study. 


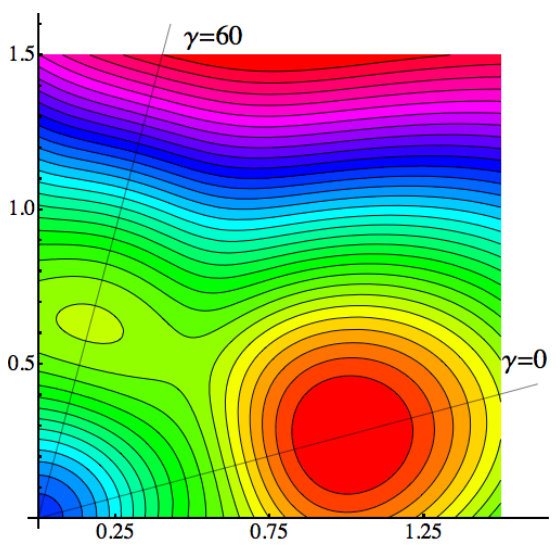

(a)

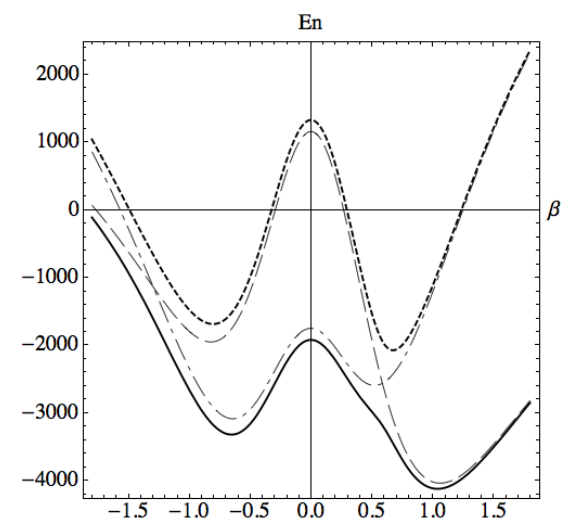

(b)

FIG. 9: Potential energy for ${ }^{182} \mathrm{Pt}$. The upper part shows the lowest eigenpotential of the matrix $E(\beta, \gamma)$ in Eq. (8). The lower part shows the potential for $\gamma=0^{\circ}$ as a function of $\beta$. Lines are as in Fig. 2 .

\section{CONCLUSIONS}

In this paper we have shown that the matrix coherentstate method applied to the IBM with configuration mixing yields results that are in close agreement with mean- field calculations, describing at the same time the known spectroscopic properties of the Pt isotopes. We have presented results for shape phase transitions in the chain of Pt isotopes from $A=182$ to 204 . Large values of the mixing parameter can produce a PES with near- $\gamma$-instability, in spite of the fact that no $\mathrm{SO}(6)$ Hamiltonian is used explicitly. We expect that the measurement of intensities of two-nucleon transfer (one-boson transfer) reactions between the Pt isotopes should produce a strong signature for criticality, indicating the presence of shape coexistence and giving direct information on the actual mixing of configurations [29].

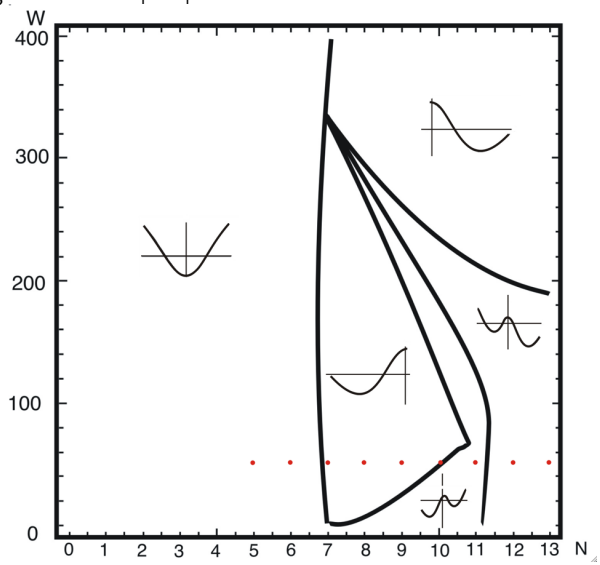

FIG. 10: The phase diagram for the lowest eigenpotential of Eq. (8) with the boson number $N$ on the $x$ axis and the mixing parameter $\omega$ on the $y$ axis.

All other parameters are taken from the Pt isotopes.

The inset figures illustrate the character of the potential

in the different regions in the diagram which are separated by critical lines The red dots correspond to the $\mathrm{Pt}$ isotopes.

\section{Acknowledgements}

This work was supported in part by CONACyT (Mexico).
[1] A.N. Andreyev et al., Nature (London) 405, 430 (2000); A.N. Andreyev et al., Nucl. Phys. A 682, 482c (2001).

[2] G.D. Dracoulis, B. Fabricius, A.E. Stuchbery, A.O. Macchiavelli, W. Korten, F. Azaiez, E. Rubel, M.A. Deleplanque, R.M. Diamond, and F.S. Stephens, Phys. Rev. C 44, R1246 (1991).

[3] P.M. Davidson, G.D. Dracoulis, T. Kibédi, A.P. Byrne, S.S. Anderssen, A.M. Baxter, B. Fabricius, G.J. Lane, and A.E. Stuchbery, Nucl. Phys. A 657, 219 (1999).

[4] F.R. May, V.V. Pashkevich, and S. Frauendorf, Phys. Lett. B 68, 113 (1977)

[5] M. Bender, P. Bonche, T. Duguet, and P.-H. Heenen, Phys. Rev. C 69, 064303 (2004).

[6] E. Caurier, G. Martinez-Pinedo, F. Nowacki, A. Poves, and A.P. Zuker, Rev. Mod. Phys. 77, 427 (2005).

[7] F. Iachello and A. Arima, The Interacting Boson Model (Cambridge University Press, Cambridge, 1987).

[8] A. Frank and P. Van Isacker, Algebraic Methods in Molecular and Nuclear Structure Physics (Wiley Interscience, New York, 1994).

[9] P.D. Duval and B.R. Barrett, Nucl. Phys. A 376, 213 (1982).

[10] K. Heyde, P. Van Isacker, M. Waroquier, J.L. Wood, and R.A. Meyer, Phys. Rep. 102, 291 (1983).

[11] J.L. Wood, K. Heyde, W. Nazarewicz, M. Huyse, and P. Van Duppen, Phys. Rep. 215, 101 (1992).

[12] K. Heyde, J. Jolie, J. Moreau, J. Ryckebusch, M. Waroquier, P. Van Duppen, M. Huyse, and J.L. Wood, Nucl. 
Phys. A 446, 189 (1987).

[13] R. Fossion, K. Heyde, G. Thiamova, and P. Van Isacker, Phys. Rev. C 67, 024306 (2003).

[14] A. Frank, O. Castaños, P. Van Isacker, and E. Padilla, in Mapping the Triangle, edited by Ani Aprahamian, Jolie A. Cizewski, Stuart Pittel, and N.Victor Zamfir, AIP Conf. Proc. No 638 (AIP, Melville, NY, 2002), p. 23.

[15] A. Frank, P. Van Isacker, and C.E. Vargas, Phys. Rev. C 69, 034323 (2004).

[16] A. Frank, P. Van Isacker, and F. Iachello, Phys. Rev. C 73, 061302(R) (2006).

[17] V. Hellemans, P. Van Isacker, S. De Baerdemacker, and K. Heyde, Nucl. Phys. A 789, 164 (2007).

[18] M.K. Harder, K.T. Tang, and P. Van Isacker, Phys. Lett. B 405, 25 (1997).

[19] K. Heyde, C. De Coster, J. Jolie, and J.L. Wood, Phys. Rev. C 46, 541 (1992).

[20] R. Gilmore, J. Math. Phys. 20, 891 (1979).

[21] J.N. Ginocchio and M.W. Kirson, Phys. Rev. Lett. 44,
1744 (1980).

[22] A.E.L. Dieperink, O. Scholten, and F. Iachello, Phys. Rev. Lett. 44, 1747 (1980).

[23] A. Bohr and B.R. Mottelson, Nuclear Structure. II Nuclear Deformations (Benjamin, New York, 1975).

[24] F. Iachello, Phys. Rev. Lett. 85, 3580 (2000).

[25] R.F. Casten and N.V. Zamfir, Phys. Rev. Lett. 85, 3584 (2000).

[26] A.E. Stuchbery, S.S. Andersen, A.P. Byrne, P.M. Davidson, G.D. Dracoulis, and G.J. Lane, Phys. Rev. Lett. 76, 2246 (1996).

[27] R. Bengtsson, T. Bengtsson, J. Dudek, G. Leander, W. Nazarewicz, and J. Zhang, Phys. Lett. B 183, 1 (1987).

[28] V. Hellemans, Shape coexistence and critical phenomena in atomic nuclei (PhD Thesis, University of Ghent, 2007).

[29] R. Fossion et al., to be published. 